\title{
Analysis of flow distribution uniformity of parallel tube sets with radial inlet
}

\author{
Si Qin ${ }^{1}$, Dongdi Liu ${ }^{2}$, Kangjia Du ${ }^{3}$, Xiaojun $\mathrm{Zhou}^{4}$ \\ School of Mechatronic Engineering and Automation, Shanghai University, \\ Shanghai, People's Republic of China \\ ${ }^{4}$ Corresponding author \\ E-mail:12503815985@qq.com, ${ }^{2} d o e r \_l d d @ 163 . c o m,{ }^{3} 741382003 @ q q . c o m,{ }^{4}$ sdzhouxj@shu.edu.cn
}

Received 11 July 2021; received in revised form 22 July 2021; accepted 29 July 2021 DOI https://doi.org/10.21595/vp.2021.22150

Check for updates

Copyright $\left({ }^{\circ} 2021\right.$ Si Qin, et al. This is an open access article distributed under the Creative Commons Attribution License, which permits unrestricted use, distribution, and reproduction in any medium, provided the original work is properly cited.

\begin{abstract}
In this paper, the flow distribution uniformity of parallel tubes with radial inlet is studied. Based on the Fluent simulation module in ANSYS WORKBENCH, the numerical simulation of parallel pipe sets is carried out, and the regularity of flow distribution uniformity of parallel pipe sets at radial inlet under different gas flow rates is obtained. In order to make the obtained law more universal, under the condition of constant simulation conditions, change the inlet position and the number of inlet, through a large number of simulation, get the flow distribution uniformity law under different conditions, and found that these laws have similarity.
\end{abstract}

Keywords: radial inlet, parallel tube set, flow distribution, FLUENT.

\section{Introduction}

Parallel tubes are commonly used in applications where uniform flow distribution is required to provide a uniform flow distribution for the target device or equipment [1]. However, the design of parallel pipe sets is mostly based on past experience rather than accurate numerical calculation or test, so it is difficult to achieve the purpose of uniform flow distribution in the initial design. Therefore, the numerical simulation of the flow distribution of parallel pipe sets can reduce the time of repeated modification of the model and provide a numerical basis for the practical use of the model [2].

In this paper, the flow distribution uniformity of a parallel tube set model with radial inlet is studied in combination with the actual operating conditions of the model. By changing the inlet position and the number of inlet, the flow distribution law of parallel tube sets with radial inlet under different model conditions was explored.

\section{Model and simulation method}

\subsection{Model and meshing}

The object studied in this paper is a parallel pipe set model with radial inlet. The pipe model is composed of straight pipe, tee, pipe cap, elbow, valve and so on [3]. In order to simplify the calculation model and reduce the amount of calculation, the model needs to be simplified. The valve at the end of the pipeline and various meters at the first section of the pipeline are ignored to obtain the calculation model in Fig. 1. In this model, the inner diameter of the pipe is $40 \mathrm{~mm}$, the wall thickness is $4 \mathrm{~mm}$, the length of the main intake pipe is $2633 \mathrm{~mm}$, the length of the branch intake pipe is $1114 \mathrm{~mm}$, and there are five branch intake pipes in total.

The fluid domain was extracted separately in the Fill command and imported into the MESH module in ANSYS for mesh division [4]. The overall mesh type was tetrahedral. After grid independence verification and comprehensive consideration of calculation time and other factors, the cell size was selected as $4 \mathrm{~mm}$. The number of grids obtained by the final grid division is 1427048 and the number of nodes is 296088 . The geometric model after meshing is shown in Fig. 2. 
In order to describe the outlet flow more conveniently and clearly, each part of the pipeline needs to be named: the branch intake pipeline and the outlet are named successively from the bottom right to the top left, as shown in Fig. 3.

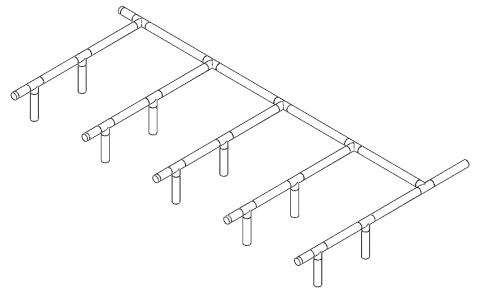

Fig. 1. Simplified pipeline model

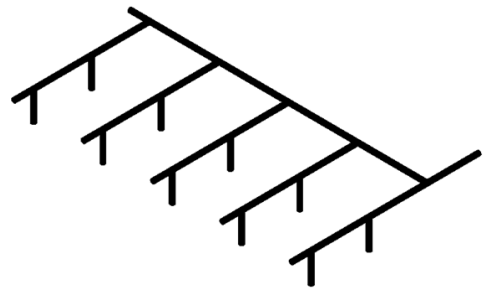

Fig. 2. Mesh division model

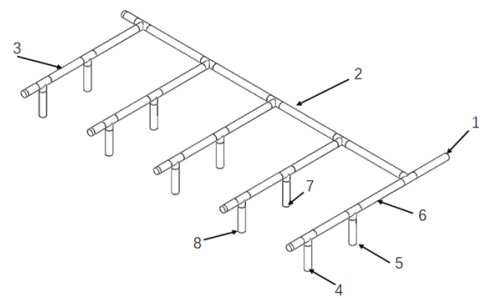

Fig. 3. Naming of each part of the model: 1 - inlet; 2 -inlet manifold; 3 - branch inlet pipe; 4 - outlet $2 ; 5$ - outlet $1 ; 6$ - branch inlet pipe $1 ; 7$ - outlet $3 ; 8$ - outlet 4

\subsection{Model hypothesis and boundary condition setting}

In this paper, the flow uniformity of parallel tube group is studied. According to the actual use conditions, the flow uniformity of this model is simulated. The following assumptions were made during the study:

1. The total intake pipe and the branch intake pipe are equally straight pipes, and the pipe diameter does not change in the simulation process except for special instructions;

2. The fluid is air and incompressible Newtonian fluid;

3. There is no temperature exchange in the process of fluid movement, and all the entering gases are of the same nature;

4. The coefficient of friction is constant throughout the pipe [5].

Boundary condition setting:

1. Inlet boundary condition.

In engineering, the purpose of adjusting the flow rate is generally achieved by controlling the mass flow rate, so the inlet boundary condition use Mass-Flow-inlet [6], and the direction is perpendicular to the inlet.

2. Outlet boundary.

The outlet of the pipeline model is a closed cavity, and the Pressure in the cavity is 1000-2000 Pa. Therefore, the outlet boundary condition use pressure-outlet [7], and the pressure is $1000 \mathrm{~Pa}$.

3. Wall boundary [8].

The wall shear of the pipeline wall adopts the wall boundary condition without slip, the airflow velocity at the pipeline wall is zero, which also conforms to the actual movement of the airflow in the pipeline.

4. Calculation model.

The turbulence calculation model adopts the Standard $k-\varepsilon$ model [9], the calculation method adopts Simple, Green-Gauss Node Based, Standard initialization, and other setting conditions adopt the default setting. When each physical quantity reaches $10^{-3}$, the calculation converges and 
the default calculation ends. Record the flow value of each outlet at this time.

Governing equation of Standard $k-\varepsilon$ model [10]:

$\frac{\partial(\rho k)}{\partial t}+\frac{\partial\left(\rho k u_{i}\right)}{\partial x_{i}}=\frac{\partial}{\partial x_{j}}\left[\left(v+\frac{v_{i}}{\sigma_{k}}\right) \frac{\partial k}{\partial x_{i}}\right]+G_{k}+G_{b}-\rho \varepsilon-Y_{M}$,
$\frac{\partial(\rho \varepsilon)}{\partial t}+\frac{\partial\left(\rho \varepsilon u_{i}\right)}{\partial x_{i}}=\frac{\partial}{\partial x_{j}}\left[\left(v+\frac{v_{i}}{\sigma_{\varepsilon}}\right) \frac{\partial \varepsilon}{\partial x_{i}}\right]+C_{1 \varepsilon} \frac{\varepsilon}{k}\left(G_{k}+C_{3 \varepsilon} G_{b}\right)-C_{2 e} \rho \frac{\varepsilon^{2}}{k}$.

\section{Simulation results and analysis of results}

\subsection{The evaluation criteria of the results}

The non-uniformity coefficient $\eta_{o i}$ of outlet flow is defined as the ratio of the mass flow of each outlet pipeline to the average value of the mass flow of each outlet pipeline, which can be expressed by the formula:

$\eta_{o i}=\frac{m_{o i}}{\bar{m}_{o}}$

In the formula, $m_{o i}$ is the mass flow rate of each outlet pipeline, and $\bar{m}_{o}$ is the average value of the mass flow rate of each outlet pipeline, the unit is $\mathrm{kg} / \mathrm{s}$.

The flow non-uniformity coefficient $\eta_{l i}$ is defined as the ratio of the mass flow of each intake pipeline to the average of the mass flow of each intake pipeline. The formula can be expressed as:

$\eta_{l i}=\frac{m_{l i}}{\bar{m}_{l}}$

In the formula, $m_{l i}$ is the mass flow of each intake pipe, and $\bar{m}_{l}$ is the average of the mass flow of each intake pipe, the unit is $\mathrm{kg} / \mathrm{s}$.

\subsection{Effect of gas velocity on uniformity of flow distribution}

In engineering applications, the mass flow rate is usually controlled to control the amount of gas entering, and under the condition of gas density and pipeline area unchanged, the change of mass flow rate is shown as the change of gas velocity. In the premise of the model unchanged, select the flow of $0.01,0.02,0.03$ and so on, a total of seven kinds of mass flow rate (unit: $\mathrm{kg} / \mathrm{s}$ ) for simulation. With the serial number of the branch intake pipeline as the abscissa and the flow nonuniformity coefficient as the ordinate (as shown in Fig. 4), the influence of gas velocity on the flow distribution uniformity was explored.

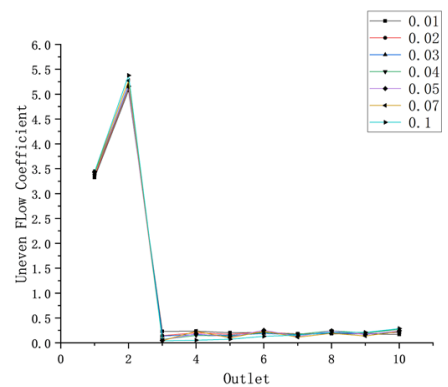

Fig. 4. Influence of flow velocity on flow non-uniformity coefficient at each outlet

Combined with the flow diagram and velocity cloud diagram in CFD-POST, it can be found 
that when the gas enters from the air inlet, a large amount of gas flows out of the air inlet pipe 1, and the gas velocity flowing to the other four air inlet pipes decreases rapidly, and the gas velocity at the end of the pipe is close to zero. This phenomenon becomes more obvious with the increase of the mass flow rate of the gas.

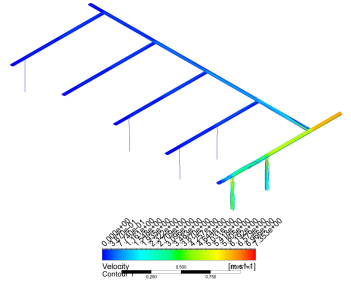

a) 0.01

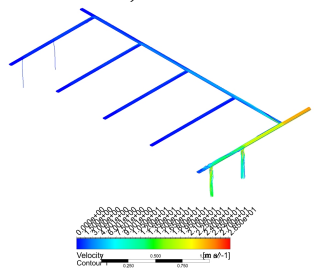

d) 0.04

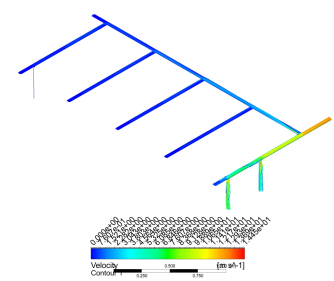

b) 0.02

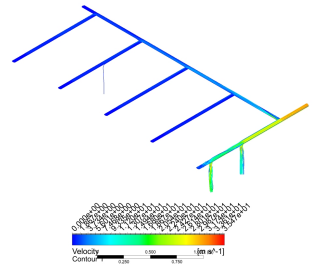

e) 0.05

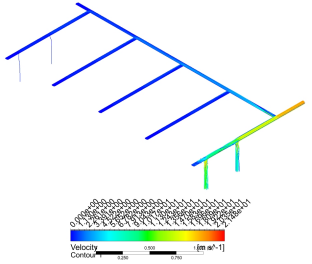

c) 0.03

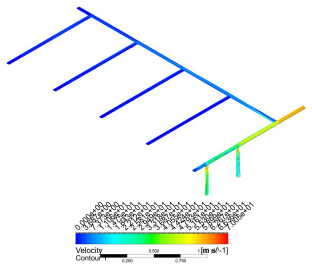

f) 0.1

Fig. 5. Velocity nephogram and streamline distribution under various flow conditions

With the increase of the mass flow rate of gas, under the condition of a certain inlet area, the entering speed of gas will be higher, and more gas will flow out of the pipeline connected with the inlet in a shorter time. When the high-speed airflow flows through a shunt tee, the sudden increase of local resistance will lead to the increase of frictional resistance and the decrease of gas velocity. According to the empirical formula, when the gas flow rate is higher, the resistance loss is greater, and the speed will decline faster, resulting in the decrease of the amount of gas flowing to the end of the pipe, and the uneven distribution of the flow of gas in other inlet pipes.

Add the outlet flow in each intake pipe, and Fig. 6 is obtained. It can be found from the analysis of Fig. 6 that the flow non-uniformity coefficient of gas in the first intake pipe is far more than 1, while the flow non-uniformity coefficient of other intake pipes fluctuates greatly due to the phenomenon of backflow.

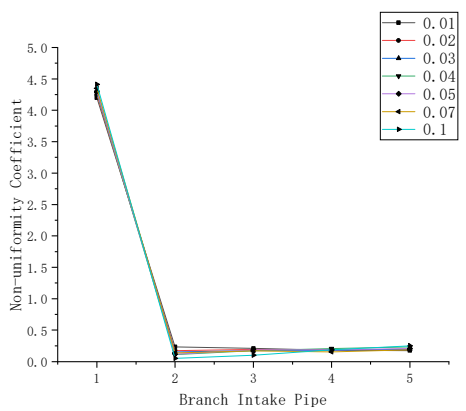

Fig. 6. Influence of flow velocity on flow non-uniformity coefficient at each branch inlet pipe

\subsection{Influence of different inlet positions on flow distribution uniformity}

After adjusting the position of the air inlet, the simulation test was carried out again. The flow of each air outlet obtained was added according to the branch intake pipe, and Fig. 7 was obtained. Where Fig. 7(a) and 7(c) were the relation diagram of the flow non-uniformity coefficient and the 
branch intake pipe, Fig. 7(b) and 7(d) are the corresponding models of Fig. 7(a), 7(c).

By analyzing a and $c$, it can be found that no matter where the air inlet is in the pipeline, most of the gas entering from the air inlet will flow out of the branch air inlet connected with the air inlet. Compared with the model in which the air inlet is connected to the first air inlet, the model in which the air inlet is connected to the third air inlet has a better flow distribution uniformity, but it is far from the ideal state.

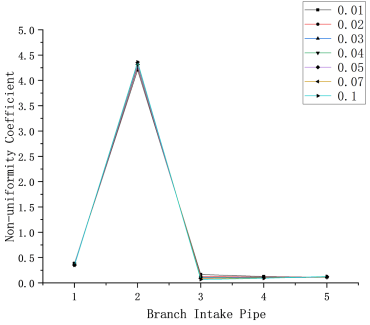

a)

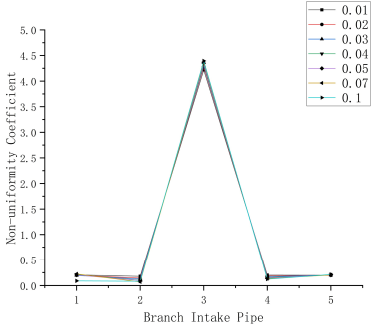

c)

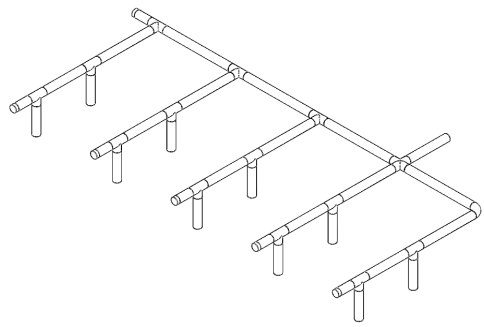

b)

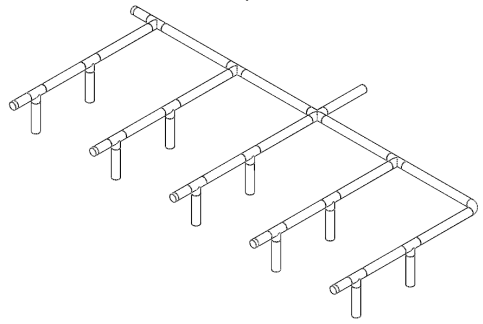

d)

Fig. 7. Influence of different inlet positions on flow distribution uniformity

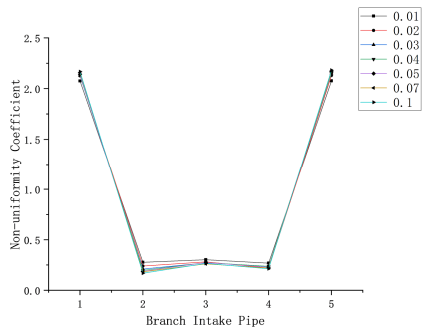

a)

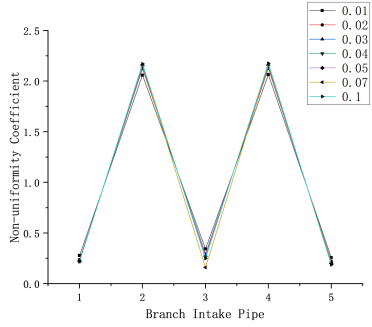

c)

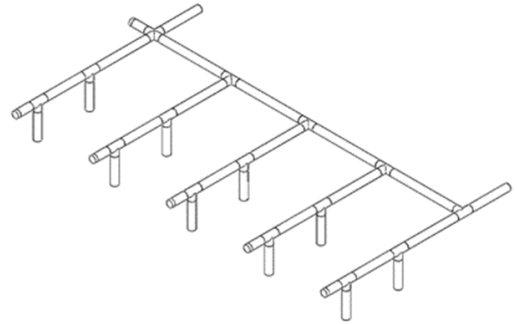

b)

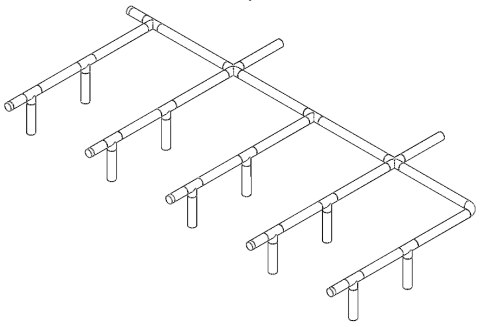

d)

Fig. 8. Influence of different number of air intakes on flow heterogeneity

\subsection{Influence of different intake numbers on flow distribution uniformity}

The number and position of air intakes were adjusted for simulation analysis again, and the flow of each air outlet was added according to the branch and air inlet pipes, and Fig. 8 was obtained. Where Fig. 8(a) and 8(c) were the relation diagram of the flow non-uniformity 
coefficient and the branch intake pipe, Figs. $8(\mathrm{~b})$ and $8(\mathrm{~d})$ are the corresponding models of Fig. 8(a) and $8(\mathrm{c})$.

Analyze Fig. 8(a) and 8(c), it can be found that although the number of air intakes is increased, the uneven flow distribution has not been greatly improved in essence. The uneven flow distribution coefficient of the branch intake pipeline connected with the air inlet is still much higher than that of other branch intake pipelines. Compared with the parallel pipe set model with only one air inlet, the parallel pipe set model with two air intakes can improve the uneven flow distribution phenomenon to a certain extent.

\section{Conclusions}

According to the above data analysis, the following conclusions can be drawn:

1) No matter how to adjust the inlet position of the parallel pipe group of the radial inlet, the flow distribution cannot be made more uniform, and the unevenness can only be improved to a certain extent. Most of the gas flows out through the branch inlet pipe connected to the inlet.

2) In engineering applications, if conditions permit, the use of parallel pipe groups with radial inlets should be avoided. When the use of parallel pipe groups with radial inlets cannot be avoided, the pipe diameters of each outlet should be appropriately adjusted to make the flow distribution more even.

3) In order to make the flow more uniform, the diameter of the inlet pipe can be appropriately increased to reduce the flow rate of the inlet pipe. Or adjust the position of the inlet pipe so that it is no longer directly connected to the branch inlet pipe, and the uniformity of its flow distribution can be appropriately changed at this time.

\section{References}

[1] J. P. Chiou, "The effect of nonuniform fluid flow distribution on the thermal performance of solar collector," Solar Energy, Vol. 29, No. 6, pp. 487-502, 1982, https://doi.org/10.1016/0038092x(82)90057-3

[2] A. Ning, J. Fan, and J. B. Ji, "An overview of CFD and PIV application in investigation of solar thermal systems," (in Chinese), Journal of Chemical Industry and Engineering Progress, No. 4, pp. 513-518, 2007.

[3] H. M. Dbouk, H. Hayek, and K. Ghorayeb, "Modular approach for optimal pipeline layout," Journal of Petroleum Science and Engineering, Vol. 197, No. 8, p. 107934, Feb. 2021, https://doi.org/10.1016/j.petrol.2020.107934

[4] L. Su and R. K. Agarwal, "CFD simulation of a supersonic steam ejector for refrigeration application," in ASME 2016 Fluids Engineering Division Summer Meeting collocated with the ASME 2016 Heat Transfer Summer Conference and the ASME 2016 14th International Conference on Nanochannels, Microchannels, and Minichannels, Jul. 2016, https://doi.org/10.1115/fedsm2016-7614

[5] R. L. Zhang and Y. H. Fang, "Analysis of flow uniformity of parallel tube group model," (in Chinese), Journal of Tianjing University of Science and Technology, Vol. 22, No. 4, pp. 45-48, 2007.

[6] N. L. Scuro et al., "RANS-based CFD calculation for pressure drop and mass flow rate distribution in an MTR fuel assembly," Nuclear Science and Engineering, Vol. 195, No. 4, pp. 349-366, Apr. 2021, https://doi.org/10.1080/00295639.2020.1825306

[7] M. Alizdeh, "Three dimensional numerical (3D CFD) study of effect of pressure-outlet and pressurefar-field boundary conditions on heat transfer predictions inside vortex tube," Journal of Progress in Solar Energy and Engineering Systems, Vol. 2, No. 1, pp. 21-25, 2018.

[8] M. H. Hosni, H. W. Coleman, and R. P. Taylor, "Measurement and calculation of fluid dynamic characteristics of rough-wall turbulent boundary-layer flows," Journal of Fluids Engineering, Vol. 115, No. 3, pp. 383-388, Sep. 1993, https://doi.org/10.1115/1.2910150

[9] T. Defraeye, B. Blocken, and J. Carmeliet, "CFD analysis of convective heat transfer at the surfaces of a cube immersed in a turbulent boundary layer," International Journal of Heat and Mass Transfer, Vol. 53, No. 1-3, pp. 297-308, Jan. 2010, https://doi.org/10.1016/j.ijheatmasstransfer.2009.09.029

[10] M. P. Schwarz and W. J. Turner, "Applicability of standard k- $\varepsilon$ turbulence model to gas stirred bath," Journal of Applied Mathematical Modelling, Vol. 12, No. 3, pp. 273-279, 1988. 